\title{
Compressed Sensing Based 3D Tomographic Reconstruction for Rotational Angiography
}

\author{
Hélène Langet ${ }^{1,2,3}$, Cyril Riddell ${ }^{1}$, Yves Trousset ${ }^{1}$, Arthur Tenenhaus ${ }^{2}$, \\ Elisabeth Lahalle ${ }^{2}$, Gilles Fleury ${ }^{2}$, and Nikos Paragios ${ }^{3}$ \\ 1 GE Healthcare, 78530 Buc, France \\ 2 Supélec, Department of Signal Processing and Electronic Systems, 91192 \\ Gif-sur-Yvette, France \\ 3 Ecole Centrale Paris, Applied Mathematics and Systems Department, 92290 \\ Châtenay-Malabry, France
}

\begin{abstract}
In this paper, we address three-dimensional tomographic reconstruction of rotational angiography acquisitions. In clinical routine, angular subsampling commonly occurs, due to the technical limitations of C-arm systems or possible improper injection. Standard methods such as filtered backprojection yield a reconstruction that is deteriorated by sampling artifacts, which potentially hampers medical interpretation.

Recent developments of compressed sensing have demonstrated that it is possible to significantly improve reconstruction of subsampled datasets by generating sparse approximations through $\ell_{1}$-penalized minimization. Based on these results, we present an extension of the iterative filtered backprojection that includes a sparsity constraint called soft background subtraction.

This approach is shown to provide sampling artifact reduction when reconstructing sparse objects, and more interestingly, when reconstructing sparse objects over a non-sparse background. The relevance of our approach is evaluated in cone-beam geometry on real clinical data.
\end{abstract}

\section{Introduction}

Rotational angiography using C-arm based X-Ray systems provides tomographic acquisitions (scan) of two-dimensional (2D) cone-beam X-Ray projection views, which are used to reconstruct a three-dimensional (3D) model of the human vasculature. To increase the contrast of vessels, a radio-opaque contrast medium must be injected into the blood. Ideally, the injection should be such that all vessels located inside the field of view are fully opacified from the beginning to the end of the contrast scan. The contrast medium being rapidly flushed in the blood flow, the rotation speed of the C-arm must be set as high as safely possible to minimize contrast use, while the detector acquisition frame rate then limits the total number of views, resulting in angular subsampling. Subsampling has little incidence on visualization of highly contrasted structures, but the smaller vessels are affected by streak artifacts originating from intense vessels as well as bone structures. Furthermore, it is common in the clinical practice that opacification

G. Fichtinger, A. Martel, and T. Peters (Eds.): MICCAI 2011, Part I, LNCS 6891, pp. 97-104, 2011. (C) Springer-Verlag Berlin Heidelberg 2011 
occurs later than expected, and thus later than the start of the C-arm rotation, resulting in inconsistently opacified projection views. If the reconstruction is performed on the subset of consistent projections, the vascular structures are then subsampled in a way known as a limited-angle problem.

Depending on the anatomy, it might be advantageous to perform two acquisitions in a single protocol, known as digital subtracted rotational angiography (DSRA), similarly to 2D digital subtracted angiography: a first scan (mask) is performed without contrast injection, while a second scan (contrast) is performed after injection. All background structures (such as bones) are removed by digital log-subtraction of the mask views from the contrast views so that a vessel-only 3D reconstruction can be performed. Because the distribution of vessels can be considered sparse, we propose to address the reconstruction of such subsampled data by using recent compressed sensing results [2, 5. These results have highlighted the interest of casting the reconstruction problem as an optimization problem constrained with a suitable sparse regularization. It has been shown in 2 that perfect reconstruction of a piecewise constant image is achievable through minimization of its total variation (TV). Several works, among which [9] and references within, have focused on the reconstruction of piecewise constant approximations of more clinically relevant type of objects, showing streak artifact reduction, but at the expense of an overall change in the image appearance, that may not be clinically acceptable.

In the case of naturally sparse image such as a vessel tree, the direct use of the image $\ell_{1}$-norm fits the compressed sensing approach without inducing an approximation, and thus is expected to provide streak reduction without changing the nature of clinical images. Still, the resulting iterative procedure for solving the optimization problem may depart from the standard image quality known in the clinical practice. It is indeed a key motivation for using alternative iterative schemes [10]. Nevertheless, in this work, we seek to only address subsampling while deviating as little as possible to the standard filtered backprojection (FBP) type of images. To this aim, we develop an extension of the iterative FBP (iFBP) that includes sparsity constraints. To our knowledge, $\ell_{1}$-regularization of iFBP has never been proposed thus far. A new algorithm, called iFBP with soft background subtraction (SBS), is derived and applied to subsampled subtracted clinical scans. It is shown to provide the expected benefits of streak reduction, in a superior way to what can be achieved applying a positivity constraint. More interestingly, it is further shown that the proposed methodology does also apply to the more general case of sparse structures over a non sparse background, where the positivity constraint is irrelevant.

\section{Method}

Let $f \in \mathbb{R}^{K}$ be the vector containing the volume, where $K$ is the number of voxels. Let $p \in \mathbb{R}^{I}$ be the vector containing the projections, where $I$ is the total number of measurements. Let $R \in \mathbb{R}^{I \times K}$ be the matrix describing the scan trajectory. The reconstruction problem that we address consists in recovering $f$ given the data constraint $p=R f$. 


\subsection{Algorithm}

A reconstruction method widely used in practice consists of a discretization of the analytical inverse of the system, i.e. backprojection of the filtered projections: $f=R^{T} D p$, where $R^{T}$ is the transpose of $R$, i.e. backprojection, and $D$ is the ramp filter. Cone-beam dedicated FBP is referred to as the Feldkamp algorithm 6]. Iterative FBP minimizes the Euclidean distance $Q$ between $p$ and the projections of $f$ filtered by $D^{\frac{1}{2}}$ that is expressed in the Fourier domain as the square root of the ramp filter and is such that $D^{\frac{1}{2}} D^{\frac{1}{2}}=D[8]$ :

$$
Q(f)=\frac{1}{2}\left\|D^{\frac{1}{2}}(R f-p)\right\|_{2}^{2}
$$

$\mathrm{iFBP}$ is a fast converging iterative reconstruction algorithm that provides advantages over FBP in handling the cone-beam geometry.

The compressed sensing theory provides a way to recover a signal from a small number of measurements if it is sparse in some basis, that is, if most of the signal energy is concentrated in a few coefficients only. In practice, minimization of the $\ell_{1}$-norm under a data constraint promotes sparsity [5] and can generate sparse approximations of undersampled signals. To get sparse approximations in tomography, we define the functional $J$ as the sum of the previous quadratic data fidelity term $Q$ and a sparsity penalty $\varphi$ :

$$
J(f)=Q(f)+\varphi(f)
$$

To minimize $J$ despite the non-differentiablity of $\varphi$, we use a simple implementation that consists of an explicit gradient step with step $\tau>0$ for minimizing $Q$, which here corresponds to an iFBP iteration, followed by an implicit step for applying constraint $\varphi$ :

$$
\left\{\begin{array}{l}
f^{\left(n+\frac{1}{2}\right)}=f^{(n)}-\tau \nabla Q\left(f^{(n)}\right) \\
f^{(n+1)}=\operatorname{prox}_{\tau, \varphi}\left(f^{\left(n+\frac{1}{2}\right)}\right)
\end{array}\right.
$$

The penalization is applied via its proximity operator which is defined as:

$$
\forall x \in \mathbb{R}^{K}, \quad \operatorname{prox}_{\gamma, \varphi}: x \rightarrow \underset{y \in \mathbb{R}^{K}}{\operatorname{argmin}}\left[\gamma \varphi(y)+\frac{1}{2}\|x-y\|_{2}^{2}\right]
$$

where $\gamma>0$ and is set equal to the gradient step $\tau$ in (3).

Note that replacing the sparsity constraint $\varphi$ by the indicator function: $\varphi(f)=$ $\left\{\begin{array}{c}0 \text { if } f \in \mathbb{R}_{+}^{K} \\ +\infty \text { otherwise }\end{array}\right.$, where $\mathbb{R}_{+}^{K}$ is the convex set of positive images, is equivalent to taking as proximity operator the projector over $\mathbb{R}_{+}^{K}$. Thereby, the scheme presented in (2) also includes the application of the positivity constraint to the iFBP algorithm. We refer the reader to 4 for a complete overview of proximal splitting methods in signal processing. 


\subsection{Sparsity Constraints}

The proximity operator associated to the image $\ell_{1}$-norm: $\varphi(f)=\lambda\|f\|_{1}$ is the soft-thresholding operator $\mathrm{S}_{\lambda \tau}(f)$ of threshold $\lambda \tau$, where $\lambda \geq 0$ is the regularization parameter that controls the strength of the $\ell_{1}$-penalization. Softthresholding is not a filter, but a segmentation step, which does not modify the image appearance above the given threshold.

This sparsity constraint can be combined with the positivity constraint in a single proximity operator that we call soft background subtraction. It is defined

by: $S B S_{\lambda \tau}(f)=\left\{\begin{array}{cc}f-\lambda \tau & \text { if } f \geq \lambda \tau \\ 0 & \text { if } f<\lambda \tau\end{array}\right.$. It promotes sparsity by removing at each iteration all structures whose intensity is lower than threshold $\lambda \tau$. Considering the case of a sparse image that only contains vessels, there is no strictly positive $\lambda$ value that may not remove some part of the vessels, and thus biase the reconstructed result.

Consequently, our approach includes defining a set of decreasing $\lambda$ values: $\Lambda=$ $\left\{\lambda_{n} \mid n=1, \cdots, N\right\}$, such that $\lambda_{1} \geq \cdots \geq \lambda_{N}=0$, where $N$ is the total number of iterations. This strategy is compatible with processing sparse structures over a non-sparse background since the background is reintroduced as $\lambda$ is decreased.

\subsection{Two-Pass Reconstruction}

We propose a simple extension of SBS dedicated to the special case of late vessel opacification in the non-subtracted case. To this purpose, we assume that opacification is a binary phenomenon and split the full scan into two subsets: the first subset contains the projection views acquired without opacification, while the second one contains the projection views acquired with opacification. SBS reconstruction is performed on each subset. These two reconstructions taken independently yield limited angular reconstruction of the non-sparse background, but once averaged can recover all background structures.

\section{Experiments}

We evaluated the reconstruction quality of the proposed algorithm on undersampled acquisitions as found in the clinical practice, where improvement over the standard reconstruction would be welcome. Data are acquired on an Innova 3100 C-arm system (GE Healthcare, Chalfont St. Giles, UK). The system records cone-beam projections at 30 frames/s during an approximately $200^{\circ}$ rotation at $40^{\circ} / \mathrm{s}$ delivering 150 views in total. iFBP algorithm was used with $\mathrm{N}=20$ iterations and a gradient step $\tau=0.95$. Direct comparison of iFBP and FBP is difficult because iFBP corrects for cone-beam artifacts and may produce images with slightly higher resolution. Consequently, in the absence of a ground truth, iFBP with positivity constraint is a better reference than FBP, from which establish SBS advantages. For SBS, $\lambda_{n}$ was initialized at $\lambda_{1}$ equal to $90 \%$ of the maximum value of the FBP reconstruction and linearly decreased to $\lambda_{N}=0$. 
For all reconstructions, a volume of $256^{3}$ voxels was computed. Intensities are given in positive Hounsfield Unit (HU), i.e air is 0 instead of $-1000 \mathrm{HU}$.

The first dataset is a DSRA exam of cerebral vessels. A subtracted scan was computed by subtraction of the mask scan from the contrast scan. During the acquisition of the contrast scan, the right vertebral artery (RVA) did not appear opacified during the first half of the rotation. Figure1 focuses on RVA subtracted reconstruction (note that RVA is the most left vessel in the slices). RVA late opacification resulted in a FBP reconstruction (Fig. 1a) that was deteriorated by horizontal streaks due to the lack of opacification in the lateral views. In addition, the RVA measured intensity (peak: $4540 \mathrm{HU}$ ) was half that of the left vertebral artery (LVA, peak: $9929 \mathrm{HU}$ ) which was seen fully opacified during the whole scan. The constrained iFBP reconstructions (Fig. 1b for positivity and Fig. 1c for SBS) recovered the RVA from the subset of fully opacified projections (75 views from 100 to $200^{\circ}$ ) both in terms of shape and intensity (RVA peak: $7541 \mathrm{HU}$ with positivity and $9697 \mathrm{HU}$ with SBS).
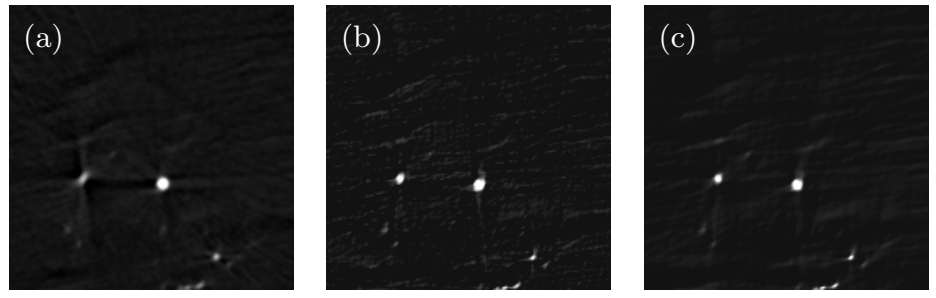

Fig. 1. Subtracted RVA (HU range: -1000 to 6000). (a) FBP reconstruction of 150 views over $0-200^{\circ}$. (b) iFBP reconstruction of 75 views over $100-200^{\circ}$ with positivity constraint. (c) iFBP reconstruction of 75 views over $100-200^{\circ}$ with SBS.

Figure 2 displays a slice higher in the brain, which was not affected by opacification delays and thus reconstructed from the full subtracted scan. Both the positivity constraint (Fig. 2b) and SBS (Fig. 2c) promoted sparsity of the reconstructed structures, thus reducing streak artifacts with respect to the FBP reconstruction of Fig. 2a However, one sees that the subtracted volume does not contain vessels only, but also perfused tissues (grey areas surrounding the vessels). These areas are not sparse, which shows the importance of an unbiased reconstruction. We computed the mean and standard deviation in a region of interest of 930 voxels within these perfused tissues and found $116 \pm 142 \mathrm{HU}$ in Fig. $2 \mathrm{~b}$ for positivity and $96 \pm 55 \mathrm{HU}$ in Fig. 2c for SBS, a twofold Signal-to-NoiseRatio (SNR) increase for SBS over positivity (1.8 vs. 0.8), which is confirmed by visual analysis of the respective slices. In terms of resolution, profile curves (not shown) drawn through vessels revealed no differences.

The second dataset is a single scan (no mask) of opacified carotid arteries. Figure 3 compares iFBP with positivity after $N=5$ and $N=20$ iterations (Fig. 3a and Fig. 3b respectively) to iFBP with SBS (Fig. 3c). Removal of the strong horizontal streaks due to a tooth metallic implant could not be obtained after 5 

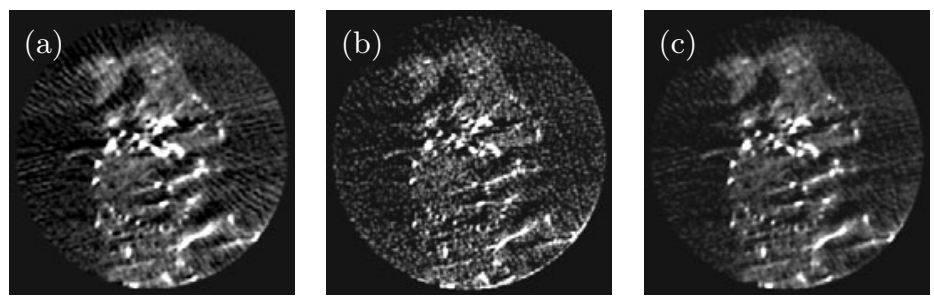

Fig. 2. Subtracted data (HU range: -100 to 500). (a) FBP reconstruction. (b) iFBP reconstruction with positivity constraint. (c) iFBP reconstruction with SBS.

iterations. After 20 iterations, the streaks were removed both by positivity and SBS. Computation of the mean and standard deviation in a volume of interest of 4000 voxels located in the soft tissues between the jaws and posterior to the teeth gave $803 \pm 60 \mathrm{HU}$ for Fig. 3a, $787 \pm 88 \mathrm{HU}$ for Fig. 3b and $775 \pm 49 \mathrm{HU}$ for Fig. 3c. SBS had the highest SNR (15.8 vs. 13.4 and 8.9), despite the 20 iterations. We attribute this SNR improvement to the reduction of intensity of smaller streaks in the soft tissues. Profile curves (not shown) through the bony structures of Fig. $3 \mathrm{~b}$ indicated a possibly slightly higher resolution for the positivity constraint after 20 iterations, although possibly linked to the strong noise increase. On the other hand, peak values over the profiles were not systematically higher in Fig. $3 \mathrm{~b}$ than in Fig. 3c.
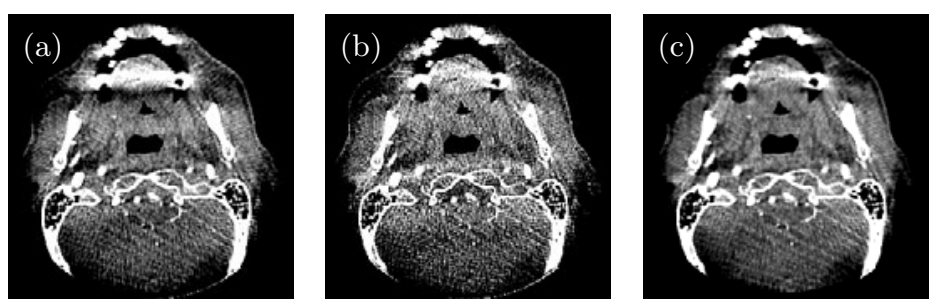

Fig. 3. Second dataset (HU range: 550 to 1050). (a) iFBP reconstruction with positivity constraint -5 iterations; (b) iFBP reconstruction with positivity constraint -20 iterations; (c) iFBP reconstruction with SBS - 20 iterations.

Application of the two-pass reconstruction is shown in Fig. 4. The same slice as in Fig. 1 was reconstructed without subtraction. Besides what we already noted in the subtracted case (Fig. 1a), the non-subtracted FBP reconstruction (Fig. 4a ) showed that the horizontal and vertical black streaks associated to the RVA hid the underlying tissues. Limitating FBP reconstruction to the second half of the scan (Fig. 4b) yielded no visual improvement of the RVA while degrading both LVA and background. On the contrary the SBS constraint (Fig. 4c) recovered both arteries, as in the subtracted case. Finally, the two-pass reconstruction was necessary to also recover the background as shown in Fig. 4d. However, the averaging operation of the two-pass reconstruction affected the quantification of 
the RVA: RVA peak value was $11744 \mathrm{HU}$ for Fig. 4c and $6353 \mathrm{HU}$ for Fig. 4d (similar to the RVA peak value of $6265 \mathrm{HU}$ for FBP). The mean and standard deviation were computed in a volume of interest of 40000 voxels located in the uniform tissues between the two arteries, yielding $1286 \pm 318 \mathrm{HU}$ for FBP (Fig. 4a) and $1070 \pm 215 \mathrm{HU}$ for the two-pass algorithm (Fig. 4d), a 25\% SNR increase for the two-pass algorithm over FBP due to streak reduction.
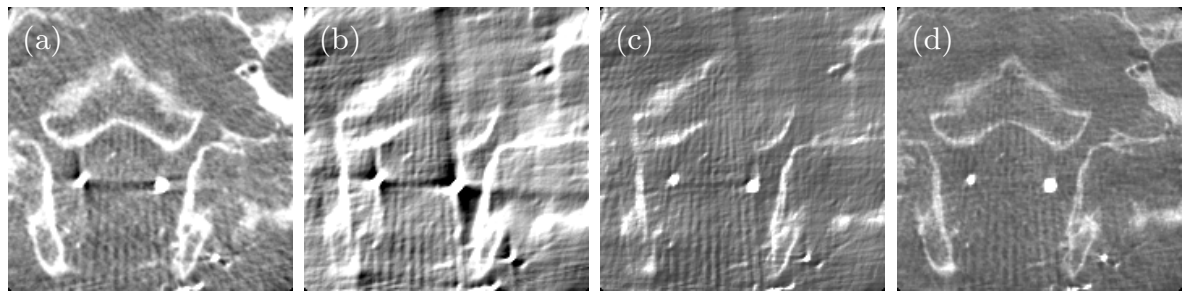

Fig. 4. Non subtracted RVA (HU range: 0 to 2400). (a) FBP reconstruction of 150 views over $0-200^{\circ}$. (b) FBP reconstruction of 75 views over $100-200^{\circ}$. (c) iFBP reconstruction of 75 views over $100-200^{\circ}$ with SBS. (d) Two-pass reconstruction.

\section{Discussion and Conclusion}

In this study, a new $\ell_{1}$-regularization of the iterative filtered backprojection, called soft background subtraction, has been introduced and applied to a selection of clinical datasets that suggests a domain of applicability: clinical cases of limited frame rate or of limited angular coverage. It has been shown to mitigate or reduce streak artifacts, when reconstructing sparse volumes from sparse data. Because we voluntarily did not model noise or other physical aspects of the imaging device beyond the cone-beam geometry, the reconstructed images had a similar appearance than FBP-type reconstructions.

The positivity constraint was effective at reducing streaks in subtracted images. Indeed, it is a special case of the proposed background subtraction technique, that can still be considered a sparse regularizer since negative voxels that get set to zero are many in this context. More interestingly, the linear decrease of the soft background subtraction threshold provided superior reconstruction of the non-sparse background of sparse high-intensity structures, resulting in more uniform tissue depiction when affected by streaks from either teeth, bones or opacified vessels, at equal resolution. Linearly decreasing the regularization strength is too simple a heuristic to exactly solve the hard-constrained compressed sensing problem, and is thus surely suboptimal. Still it has an intuitive interpretation that makes it well suited for a clinical use. Most importantly, the generated solution is not a compromise between a fitting term and a penalty, but targets an unbiased fit to the data. SBS provided to a certain extent the expected benefits of compressed sensing theory: reduced streaks. Such gains would otherwise be achievable only by increasing the number of projections, which was not possible in these clinical settings, rather than increasing the X-Ray dose of each projection image. 
The soft background subtraction approach is applicable to any tomographic dataset to provide localized and specific improvements regarding subsampling while appearing just as good as FBP otherwise. The combination of iterative FBP and simple thresholding operations entails a computation time of roughly twice the standard FBP computation per iteration. Further studies are required to determine whether the number of iterations can be reduced.

The two-pass algorithm recovered the shape of the degraded vessel with its background, but not its quantification. To recover both, we need to recast the problem as a dynamic one. Compressed sensing based tomographic reconstruction of dynamic series is an active research area [3, 7] that implies to address higher levels of undersampling than those currently seen in the clinical practice. We anticipate that the proposed approach will provide a key brick to such reconstructions, as proximal splitting allows for combining iterative FBP with a variety of non-linear filters or segmentations, that can be derived by the calculus of variations [1].

\section{References}

1. Aubert, G., Kornprobst, P.: Mathematical Problems in Image Processing: Partial Differential Equations and the Calculus of Variations. Applied Mathematical Sciences, vol. 147. Springer, Heidelberg (2006)

2. Candès, E., Romberg, J., Tao, T.: Robust Uncertainty Principles: Exact Signal Reconstruction from Highly Incomplete Frequency Information. IEEE Transactions on Information Theory 52(2), 489-509 (2006)

3. Chen, G.H., Tang, L.: Prior Image Constrained Compressed Sensing (PICCS). Medical Physics 35(2), 660-663 (2008)

4. Combettes, P.L., Pesquet, J.C.: Proximal Splitting Methods in Signal Processing. In: Bauschke, H.H., Burachik, R.S., Combettes, P.L., Elser, V., Luke, D.R., Wolkowicz, H. (eds.) Fixed-Point Algorithms for Inverse Problems in Science and Engineering, Springer Optimization and Its Applications., vol. 49, ch.10, pp. 185212. Springer, Heidelberg (2011)

5. Donoho, D.L.: For Most Large Underdetermined Systems of Linear Equations, the Minimal $\ell_{1}$ Solution is Also the Sparsest Solution. Communications on Pure and Applied Mathematics 59(6), 797-829 (2006)

6. Feldkamp, L.A., Davis, L.C., Kress, J.W.: Practical Cone-Beam Algorithm. Journal of the Optical Society of America A 1(6), 612-619 (1984)

7. Jia, X., Lou, Y., Dong, B., Tian, Z., Jiang, S.: 4D Computed Tomography Reconstruction from Few-Projection Data via Temporal Non-local Regularization. In: Jiang, T., Navab, N., Pluim, J.P.W., Viergever, M.A. (eds.) MICCAI 2010. LNCS, vol. 6361, pp. 143-150. Springer, Heidelberg (2010)

8. Riddell, C., Savi, A., Gilardi, M., Fazio, F.: Frequency Weighted Least Squares Reconstruction of Truncated Transmission SPECT Data. IEEE Transactions on Nuclear Science 43(4), 2292-2298 (1996)

9. Ritschl, L., Bergner, F., Fleischmann, C., Kachelrieß, M.: Improved Total Variation-Based CT Image Reconstruction Applied to Clinical Data. Physics in Medicine and Biology 56(6), 1545-1561 (2011)

10. Thibault, J., Sauer, K., Bouman, C., Hsieh, J.: A Three-dimensional Statistical Approach to Improved Image Quality for Multi-slice Helical CT. Medical Physics 34(11), 4526-4544 (2007) 